\title{
High harmonic generation in a gas-filled hollow-core photonic crystal fiber
}

\author{
O.H. Heckl · C.R.E. Baer · C. Kränkel · S.V. Marchese • \\ F. Schapper • M. Holler $\cdot$ T. Südmeyer $\cdot$ J.S. Robinson • \\ J.W.G. Tisch $\cdot$ F. Couny $\cdot$ P. Light $\cdot$ F. Benabid $\cdot$ U. Keller
}

Received: 28 September 2009 / Published online: 14 October 2009

(C) The Author(s) 2009. This article is published with open access at Springerlink.com

\begin{abstract}
High harmonic generation (HHG) of intense infrared laser radiation $[1,2]$ enables coherent vacuum-UV (VUV) to soft-X-ray sources. In the usual setup, energetic femtosecond laser pulses are strongly focused into a gas jet, restricting the interaction length to the Rayleigh range of the focus. The average photon flux is limited by the low conversion efficiency and the low average power of the complex laser amplifier systems [3-6] which typically operate at kilohertz repetition rates. This represents a severe limitation for many experiments using the harmonic radiation in fields such as metrology or high-resolution imaging. Driving HHG with novel high-power diode-pumped multi-megahertz laser systems has the potential to significantly increase the average photon flux. However, the higher average power comes at the expense of lower pulse energies because the repetition rate is increased by more than a thousand times, and efficient HHG is not possible in the usual geometry. So far, two promising techniques for HHG at lower pulse energies were developed: external build-up cavities $[7,8]$ and
\end{abstract}

O.H. Heckl ( $\square)$ · C.R.E. Baer · C. Kränkel · S.V. Marchese ·

F. Schapper - M. Holler · T. Südmeyer - U. Keller

Department of Physics, Institute for Quantum Electronics,

ETH Zurich, 8093 Zurich, Switzerland

e-mail: heckl@phys.ethz.ch

J.S. Robinson

Department of Physics, University of California at Berkeley and Materials Sciences Division, Lawrence Berkeley National Laboratory, Berkeley, CA 94720, USA

J.W.G. Tisch

Quantum Optics and Laser Science Group, Blackett Laboratory,

Imperial College London, London SW7 2BW, UK

F. Couny $\cdot$ P. Light $\cdot$ F. Benabid

Department of Physics, University of Bath, Bath BA2 7AY, UK resonant field enhancement in nanostructured targets [9]. Here we present a third technique, which has advantages in terms of ease of HHG light extraction, transverse beam quality, and the possibility to substantially increase conversion efficiency by phase-matching [10-14]. The interaction between the laser pulses and the gas occurs in a Kagome-type Hollow-Core Photonic Crystal Fiber (HC-PCF) [15], which reduces the detection threshold for $\mathrm{HHG}$ to only $200 \mathrm{~nJ}$. This novel type of fiber guides nearly all of the light in the hollow core [16], preventing damage even at intensities required for HHG. Our fiber guided 30-fs pulses with a pulse energy of more than $10 \mu \mathrm{J}$, which is more than five times higher than for any other photonic crystal fiber [17].

PACS 42.81.Qb - 42.65.Ky · 42.55.Xi 
In free space, the nonlinear interaction of light with gases is limited by diffraction. Guiding light in gas-filled HC-PCFs enabled several major breakthroughs in nonlinear optics. Combining a small mode area with a long effective interaction length reduces the threshold of various nonlinear processes by several orders of magnitude and increases their efficiency dramatically $[15,16,18]$. According to numerical simulations, HC-PCFs also have a large potential for high harmonic generation [11-13, 17-19]. So far, wave-guide quasi-phase matching techniques for HHG were only realized in capillaries with diameters above $100 \mu \mathrm{m}$ [20, 21]. HC-PCFs offer substantially lower transmission losses, smaller mode areas, and larger design freedom. However, employing HC-PCFs in the area of high field science is challenging, because intensities $>10^{13} \mathrm{~W} / \mathrm{cm}^{2}$ have to be achieved in the hollow core. This is difficult with standard photonic bandgap (PBG) HC-PCFs because the field intensity in surrounding silica is $\approx 1 \%$ of the maximum intensity in the core [22]. So far, the highest guided energy reported for femtosecond pulse propagation in PBG HC-PCFs is $1.8 \mu \mathrm{J}$ using 40 -fs pulses [17]. In our experiment, we used a Kagome-type HC-PCF for which the field overlap is more than ten times lower [16]. In contrast to PBG HC-PCF, the guiding mechanism is not based on a bandgap but on the inhibited coupling between the core and cladding modes [16]. The fiber is immune to core-mode and surface-mode coupling [23], which represents one of the major sources of optical overlap with the silica surrounding the fiber core. Since the damage threshold scales with the inverse of this fraction, guidance of very high pulse energies and peak power levels becomes feasible. Furthermore, Kagome-type PCFs have advantages in terms of dispersion management and an ultrabroad guiding bandwidth, which are particularly important for applications with ultrashort laser pulses [16]. A scanning electron microscope (SEM) image of our Kagome-type PCF can be seen in Fig. 1. The fiber was designed for operation at a central wavelength of $800 \mathrm{~nm}$ and has an effective core diameter of $15 \mu \mathrm{m}$.

In our experimental setup (Fig. 2), we use two vacuum chambers which are connected by a $15-\mathrm{mm}$ long HC-PCF. The driving laser is a standard Ti:sapphire amplifier system generating 30 -fs pulses at $800 \mathrm{~nm}$ with a repetition rate of $1 \mathrm{kHz}$. The entrance face of the HC-PCF is situated in the pre-chamber filled with xenon at a variable pressure of up to 30 mbar. The exit face of the fiber extended into the adjacent spectrometer vacuum chamber which is sealed off from the pre-chamber except for the gas flow through the HC-PCF and some parasitic leakage through the fiber mount. The vacuum chamber contains a tunable grating and an electron multiplier tube (EMT) detector. This iridium-coated grating has 1200 lines $/ \mathrm{mm}$, resulting in a spectral resolution of approximately $4 \mathrm{~nm}$ for our geometry. The EMT is sensitive in the spectral region from $30 \mathrm{~nm}$ to $150 \mathrm{~nm}$ with an estimated peak efficiency of $16 \%$ at a wavelength around $70 \mathrm{~nm}$.

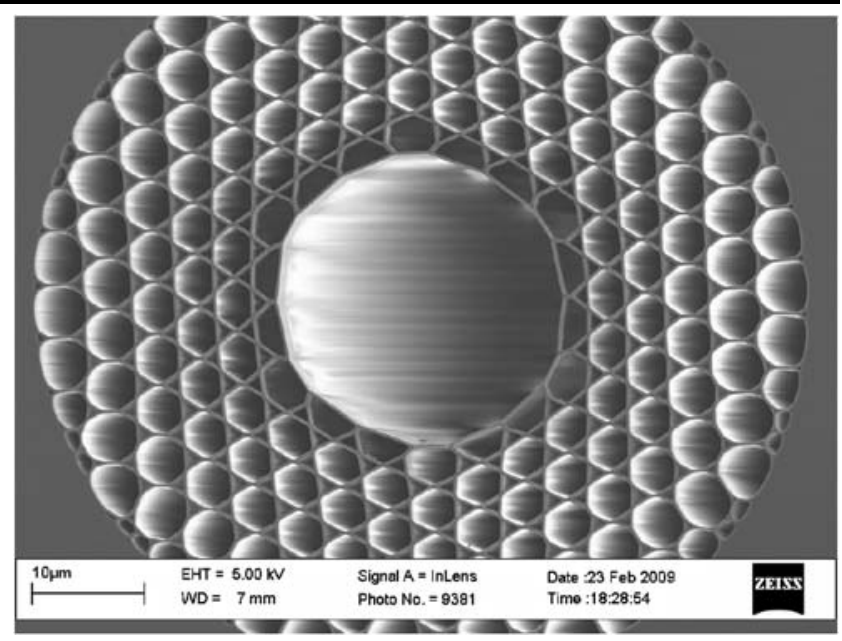

Fig. 1 SEM image of the Kagome-type HC-PCF. The effective mode diameter (corresponding to the hollow center core) is about $15 \mu \mathrm{m}$

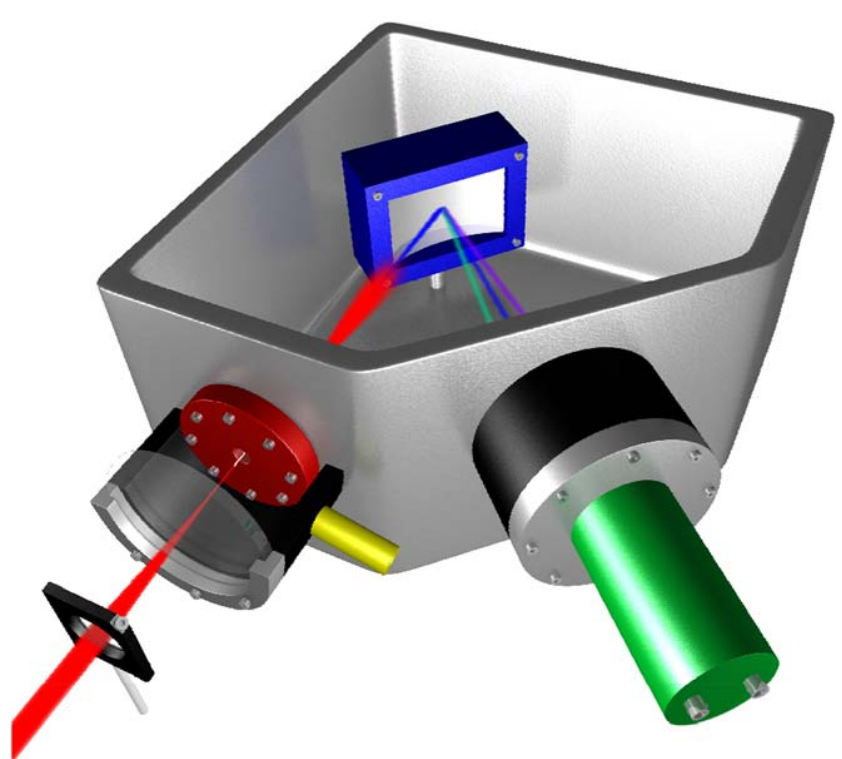

Fig. 2 Experimental setup: The laser pulses are focused onto the entrance face of the hollow core photonic crystal fiber (HC-PCF) in free space. The fiber is mounted in the middle of the red disc, which is located in a chamber backfilled with an adjustable xenon pressure. The xenon inlet is displayed in yellow. The fiber exit face is in a vacuum chamber which contains the spectrometer grating (framed in blue) and the detector (green)

As a first step, we investigated the transmission properties and damage threshold of our Kagome HC-PCF in vacuum (no xenon filling in the pre-chamber). Kagome-type fibers typically operate at high coupling efficiencies and with transmission losses of $\approx 0.5 \mathrm{~dB} / \mathrm{m}$ [16]. However, in our experiment, the input beam was elliptical and not well mode-matched to the round fiber mode, which resulted in a coupling efficiency of only $30 \%$ to $40 \%$. For a pulse-energy of $30 \mu \mathrm{J}$ incident on the fiber, we transmitted $10.5 \mu \mathrm{J}$ pulses. The spectrum at the output was identical to the input spec- 


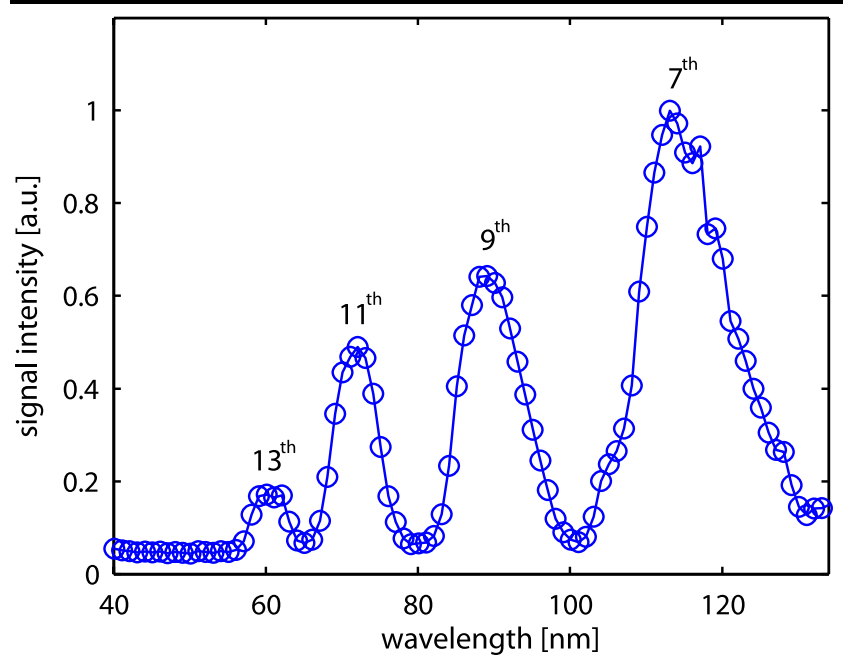

Fig. 3 Measured spectrum of the high harmonics generated in Xe with $4.2 \mu \mathrm{J}, 30 \mathrm{fs}$ laser pulses of a Ti:sapphire. The graph is corrected for spectral response of the EMT

trum, showing that the low field overlap of the core mode with the silica did not introduce significant self phase modulation. The duration of the output was not measured, but is expected to be the same as the input pulse duration due to the low dispersion of the fiber. We did not observe any damage for these pulse energies despite peak intensities of $3 \times 10^{14} \mathrm{~W} / \mathrm{cm}^{2}$ and the large amount of un-launched power.

When we flooded the pre-chamber with xenon gas, we observed the generation of high harmonics. Figure 3 shows the measured spectrum for pulses with $4.2 \mu \mathrm{J}$ energy in the HC-PCF and a xenon pressure of $P_{0}=27 \mathrm{mbar}$ in the prechamber. We did not increase the pressure above $30 \mathrm{mbar}$ because the parasitic leakage through the fiber mount degraded the vacuum in the main chamber to the EMT operation limit $\left(<1.3 \times 10^{-4} \mathrm{mbar}\right)$. The 7 th to 13 th harmonic can be clearly identified. The 3rd and 5th harmonic orders cannot be observed since the detector is insensitive to wavelengths above $140 \mathrm{~nm}$. We obtain a conversion efficiency of $\eta \approx 2 \times 10^{-9}$ (which is defined as $\eta=$ harmonic energy/launched laser energy), by integrating over the harmonic orders 7-13 and taking into account the grating efficiency (calculated with the program REFLEC [24]). Figure 4 shows the signal intensity of the 7th harmonic versus the launched pulse energy $E_{L}$ on a logarithmic plot. The harmonic signal scales as $E_{L}^{p}$ where $p=3.6$. This is a weaker dependence than expected from lowest order perturbation theory $(p \sim 7)$ indicating that the atomic response cannot be accurately described within the weak-field limit for our conditions [25]. We observed a HHG detection threshold as low as $200 \mathrm{~nJ}$ inside the HC-PCF. The transverse beam profile of the harmonics was measured by gradually inserting a knife edge into the high harmonic beam (located in vacuum chamber between fiber output and grating, see Fig. 2). In Fig. 5, we show the measurement of the beam profile for

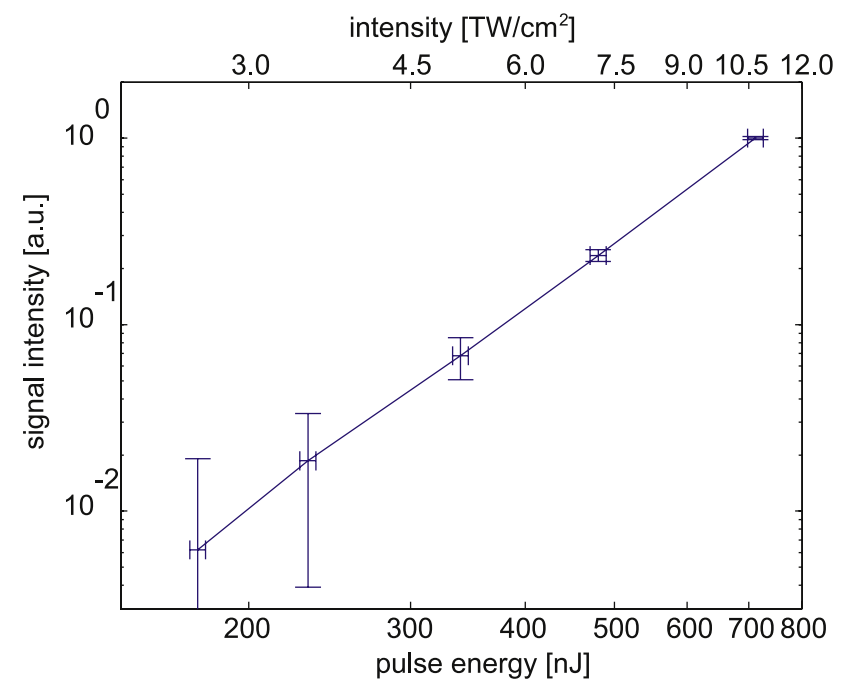

Fig. 4 Measured signal intensity for the 7th harmonic as a function of the laser pulse energy incident on the fiber. The error bars display the standard error of the measurement. The 7th HHG detection threshold was measured to be $200 \mathrm{~nJ}$ inside the HC-PCF

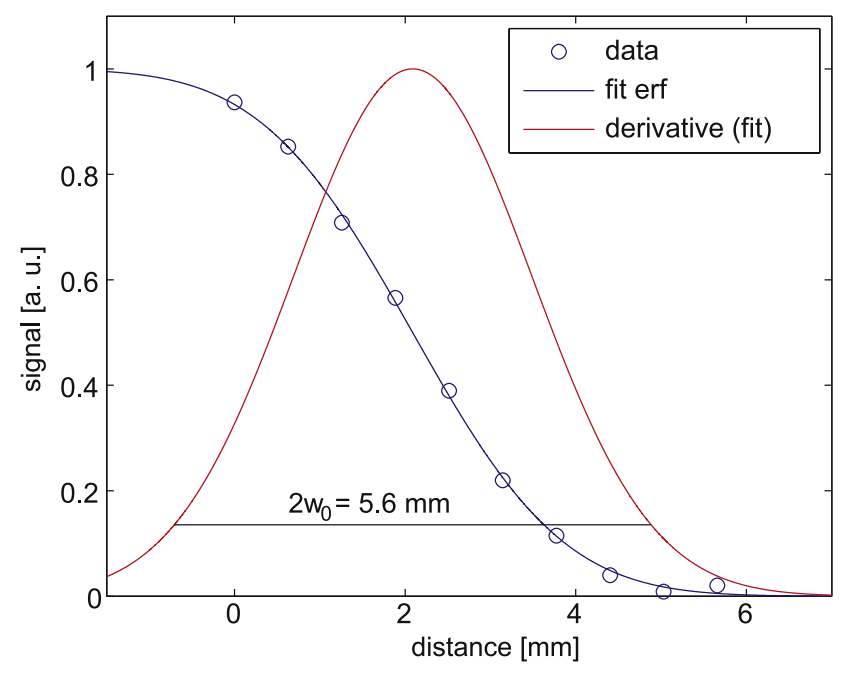

Fig. 5 A knife edge measurement of the 7th harmonic $70 \mathrm{~mm}$ from the fiber end reveals an excellent Gaussian beam profile which can be well approximated by a Gaussian fit

the 7th harmonic for a launched pulse-energy of $4 \mu \mathrm{J}$ and a xenon pressure of $30 \mathrm{mbar}$. The beam profile can be well approximated by a Gaussian fit.

Our results show that HHG in a HC-PCF is advantageous in terms of threshold, light extraction and beam quality. Extracting the harmonic radiation in enhancement cavities is substantially more challenging $[7,8]$ because the interaction of the laser pulses with the gas target occurs inside a high-finesse cavity for which the losses usually are kept well below $1 \%$. In contrast to HHG based on resonant field enhancement in nanostructured targets [9], there is no beam profile distortion imprinted onto the harmonic radia- 
tion, instead a round beam with Gaussian transverse profile is achieved (Fig. 5).

The current conversion efficiency is similar to HHG based on nanostructured targets, but substantially lower than for recent high power enhancement cavity systems [26]. This is due to the imperfect phase matching between fundamental and harmonic radiation in these first proof-ofprinciple experiments. HHG quasi-phase matching techniques are already established for interactions in capillaries [21] and free space [27], for which they increased the efficiency by more than 2 orders of magnitude. HC-PCFs allow us to address a larger parameter space for phase matching, due the substantial design flexibility of their dispersion and mode area. This is in stark contrast to standard capillaries for which the dispersion and hence phase matching conditions are directly constrained by the mode area. For example, the ability to tailor the refractive index of the fiber at the fundamental wavelength may allow for adjustment of phasematching pressure without compromising the mode area required for low energy HHG. In conjunction with the much increased interaction length afforded by the high transmission of the HC-PCF, efficient generation of high harmonics in regimes that are not limited by the absorption length of the nonlinear medium may be possible. Additionally, one can employ quasi-phase matching techniques for HHG already demonstrated in capillaries [21], for example, by modulating the mode area (e.g., by the fiber drawing method or heat treatment with a $\mathrm{CO}_{2}$ laser [28]). One may also utilize counter-propagating beams [14] as discussed in recent simulations of HHG in a PCF [11], which show that harmonics with photon energies of several hundreds of electron-volts can be produced by femtosecond pulses with microjoule energies.

In conclusion, we have demonstrated the generation of high harmonics in a gas filled HC-PCF for the first time. The fiber transmitted laser pulses with pulse energies of more than $10 \mu \mathrm{J}$ without damage, which is several times higher than previously reported for the propagation of femtosecond pulses in a HC-PCF. Using xenon as the nonlinear medium, we observed the generation of the 7th to 13th harmonic of the fundamental wavelength of a Ti:sapphire laser system operating around $800 \mathrm{~nm}$. This corresponds to the generation of light in the VUV/XUV-spectral region from $50 \mathrm{~nm}$ to $130 \mathrm{~nm}$. The observed HHG detection threshold is below $200 \mathrm{~nJ}$. This is well within reach of novel multi-megahertz diode-pumped solid-state lasers and fiber amplifiers, which achieve substantially higher power levels and repetition rates than the currently dominant kilohertz Ti:sapphire amplifier systems. Diode-pumped SESAM-mode-locked femtosecond thin disk lasers currently generate close to $100 \mathrm{~W}$ directly from the oscillator without any further amplification $[4,29,30]$. Chirped-pulse femtosecond fiber amplifier systems achieve up to $325 \mathrm{~W}$ average power [5, 6]. The resulting high average power, compact coherent XUV/VUV source will have a high impact for numerous applications in fields as diverse as medicine, biology, chemistry, physics and materials science. Even at a low conversion efficiency of only $10^{-8}$, an average HHG photon flux of $>1 \mu \mathrm{W}$ would be achieved, which is sufficient for many applications. Better conversion efficiencies with improved phase matching will reduce the power requirement and complexity of the system further. Operating at multi-megahertz instead of kilohertz repetition rates increases the signal-tonoise ratio and reduces the time required for many measurements, as, for example, in XUV metrology, high resolution XUV imaging, and microscopy [31]. Increasing the repetition rate is especially important in experiments, for which the total number of simultaneous ionization processes is limited by space charge effects (i.e., electro-magnetic forces between the generated charged particles within the interaction volume). Important examples are surface science and condensed matter studies using angle-resolved photoemission spectroscopy (ARPES) [32].

Acknowledgement The authors would like to thank Philip St. J. Russell for fruitful initial discussions on HHG in a HC-PCF and helping to set up the collaboration with Bath University.

Open Access This article is distributed under the terms of the Creative Commons Attribution Noncommercial License which permits any noncommercial use, distribution, and reproduction in any medium, provided the original author(s) and source are credited.

\section{References}

1. M. Ferray, A. L'Huillier, X.F. Li, L.A. Lompré, G. Mainfray, C. Manus, Multiple-harmonic conversion of $1064 \mathrm{~nm}$ radiation in rare gases. J. Phys. B: At. Mol. Opt. Phys. 21, L31 (1988)

2. A. McPherson, G. Gibson, H. Jara, U. Johann, T.S. Luk, I.A. McIntyre, K. Boyer, C.K. Rhodes, Studies of multiphoton production of vacuum-ultraviolet radiation in the rare gases. J. Opt. Soc. Am. B 4, 595 (1987)

3. U. Keller, Recent developments in compact ultrafast lasers. Nature 424, 831 (2003)

4. T. Südmeyer, S.V. Marchese, S. Hashimoto, C.R.E. Baer, G. Gingras, B. Witzel, U. Keller, Femtosecond laser oscillators for highfield science. Nat. Photonics 2, 599 (2008)

5. F. Röser, J. Rothhard, B. Ortac, A. Liem, O. Schmidt, T. Schreiber, J. Limpert, A. Tünnermann, $131 \mathrm{~W} 220$ fs fiber laser system. Opt. Lett. 30, 2754 (2005)

6. T. Eidam, S. Hadrich, F. Roser, E. Seise, T. Gottschall, J. Rothhardt, T. Schreiber, J. Limpert, A. Tunnermann, A 325-W-averagepower fiber CPA system delivering sub-400 fs pulses. IEEE J. Sel. Top. Quantum Electron. 15, 187 (2009)

7. C. Gohle, T. Udem, M. Herrmann, J. Rauschenberger, R. Holzwarth, H.A. Schuessler, F. Krausz, T.W. Hänsch, A frequency comb in the extreme ultraviolet. Nature 436, 234 (2005)

8. R.J. Jones, K.D. Moll, M.J. Thorpe, J. Ye, Phase-coherent frequency combs in the vacuum ultraviolet via high-harmonic generation inside a femtosecond enhancement cavity. Phys. Rev. Lett. 94, 193201 (2005) 
9. S. Kim, J.H. Jin, Y.J. Kim, I.Y. Park, Y. Kim, S.W. Kim, Highharmonic generation by resonant plasmon field enhancement. Nature 453, 757 (2008)

10. A. Paul, R.A. Bartels, R. Tobey, H. Green, S. Weiman, I.P. Christov, M.M. Murnane, H.C. Kapteyn, S. Backus, Quasi-phasematched generation of coherent extreme-ultraviolet light. Nature 421, 51 (2003)

11. H. Ren, A. Nazarkin, J. Nold, P.S.J. Russell, Quasi-phase-matched high harmonic generation in hollow core photonic crystal fibers. Opt. Express 16, 17052 (2008)

12. E.E. Serebryannikov, D. von der Linde, A.M. Zheltikov, Phasematching solutions for high-order harmonic generation in hollowcore photonic-crystal fibers. Phys. Rev. E (Stat. Nonlinear Soft Matter Phys.) 70, 66611 (2004)

13. E.E. Serebryannikov, D. von der Linde, A.M. Zheltikov, Broadband dynamic phase matching of high-order harmonic generation by a high-peak-power soliton pump field in a gas-filled hollow photonic-crystal fiber. Opt. Lett. 33, 977 (2008)

14. X. Zhang, A.L. Lytle, T. Popmintchev, X. Zhou, H.C. Kapteyn, M.M. Murnane, O. Cohen, Quasi-phase-matching and quantumpath control of high-harmonic generation using counterpropagating light. Nat. Phys. 3, 270 (2007)

15. F. Benabid, J.C. Knight, G. Antonopoulos, P.S.J. Russell, Stimulated Raman scattering in hydrogen-filled hollow-core photonic crystal fiber. Science 298, 399 (2002)

16. F. Couny, F. Benabid, P.J. Roberts, P.S. Light, M.G. Raymer, Generation and photonic guidance of multi-octave optical-frequency combs. Science 318, 1118 (2007)

17. C.J. Hensley, M.A. Foster, B. Shim, A.L. Gaeta, Extremely high coupling and transmission of high-powered-femtosecond pulses in hollow-core photonic band-gap fiber, in Conference on Lasers and Electro-Optics (CLEO) (IEEE Press, New York, 2008), p. paper JFG1

18. P.S.J. Russell, Photonic-crystal fibers. J. Lightwave Technol. 24, 4729 (2006)

19. I. Christov, H. Kapteyn, M. Murnane, Quasi-phase matching of high-harmonics and attosecond pulses in modulated waveguides. Opt. Express 7, 362 (2000)

20. D.M. Gaudiosi, B. Reagan, T. Popmintchev, M. Grisham, M. Berrill, O. Cohen, B.C. Walker, M.M. Murnane, H.C. Kapteyn, J.J. Rocca, High-order harmonic generation from ions in a capillary discharge. Phys. Rev. Lett. 96, 4 (2006)
21. E.A. Gibson, A. Paul, N. Wagner, R. Tobey, D. Gaudiosi, S. Backus, I.P. Christov, A. Aquila, E.M. Gullikson, D.T. Attwood, M.M. Murnane, H.C. Kapteyn, Coherent soft X-ray generation in the water window with quasi-phase matching. Science 302, 95 (2003)

22. G. Humbert, J. Knight, G. Bouwmans, P. Russell, D. Williams, P. Roberts, B. Mangan, Hollow core photonic crystal fibers for beam delivery. Opt. Express 12, 1477 (2004)

23. J. West, C. Smith, N. Borrelli, D. Allan, K. Koch, Surface modes in air-core photonic band-gap fibers. Opt. Express 12, 1485 (2004)

24. F. Schäfers, The BESSY raytrace program RAY, in Modern Developments in X-Ray and Neutron Optics (Springer, Berlin/Heidelberg, 2008), pp. 9-41

25. A. L'Huillier, P. Balcou, L.A. Lompre, Coherence and resonance effects in high-order harmonic-generation. Phys. Rev. Lett. 68, 166 (1992)

26. D.C. Yost, T.R. Schibli, J. Ye, Efficient output coupling of intracavity high-harmonic generation. Opt. Lett. 33, 1099 (2008)

27. J. Seres, V.S. Yakovlev, E. Seres, C. Streli, P. Wobrauschek, C. Spielmann, F. Krausz, Coherent superposition of laser-driven soft-X-ray harmonics from successive sources. Nat. Phys. 3, 878 (2007)

28. T.E. Dimmick, G. Kakarantzas, T.A. Birks, P.S.J. Russell, Carbon dioxide laser fabrication of fused-fiber couplers and tapers. Appl. Opt. 38, 6845 (1999)

29. J. Neuhaus, D. Bauer, J. Zhang, A. Killi, J. Kleinbauer, M. Kumkar, S. Weiler, M. Guina, D.H. Sutter, T. Dekorsy, Subpicosecond thin-disk laser oscillator with pulse energies of up to 25.9 microjoules by use of an active multipass geometry. Opt. Express 16, 20530 (2008)

30. F. Brunner, E. Innerhofer, S.V. Marchese, T. Südmeyer, R. Paschotta, T. Usami, H. Ito, S. Kurimura, K. Kitamura, G. Arisholm, U. Keller, Powerful red-green-blue laser source pumped with a mode-locked thin disk laser. Opt. Lett. 29, 1921 (2004)

31. W. Chao, B.D. Harteneck, J.A. Liddle, E.H. Anderson, D.T. Attwood, Soft-X-ray microscopy at a spatial resolution better than $15 \mathrm{~nm}$. Nature 435, 1210 (2005)

32. J.D. Koralek, J.F. Douglas, N.C. Plumb, Z. Sun, A.V. Fedorov, M.M. Murnane, H.C. Kapteyn, S.T. Cundiff, Y. Aiura, K. Oka, H. Eisaki, D.S. Dessau, Laser based angle-resolved photoemission, the sudden approximation, and quasiparticle-like spectral peaks in $\mathrm{Bi}_{2} \mathrm{Sr}_{2} \mathrm{CaCu}_{2} \mathrm{O}_{8}$. Phys. Rev. Lett. 96, 017005 (2006) 